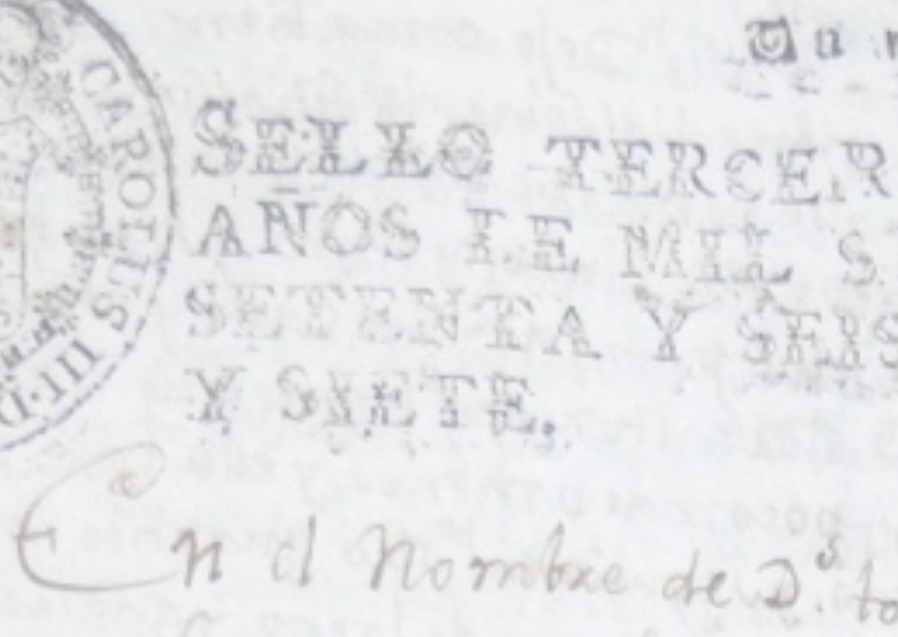

Ina Arnen. Wepown quant:" eitar Cax mirnexa Voluntar Viexen como huxal de eita Andad delexio Bribil

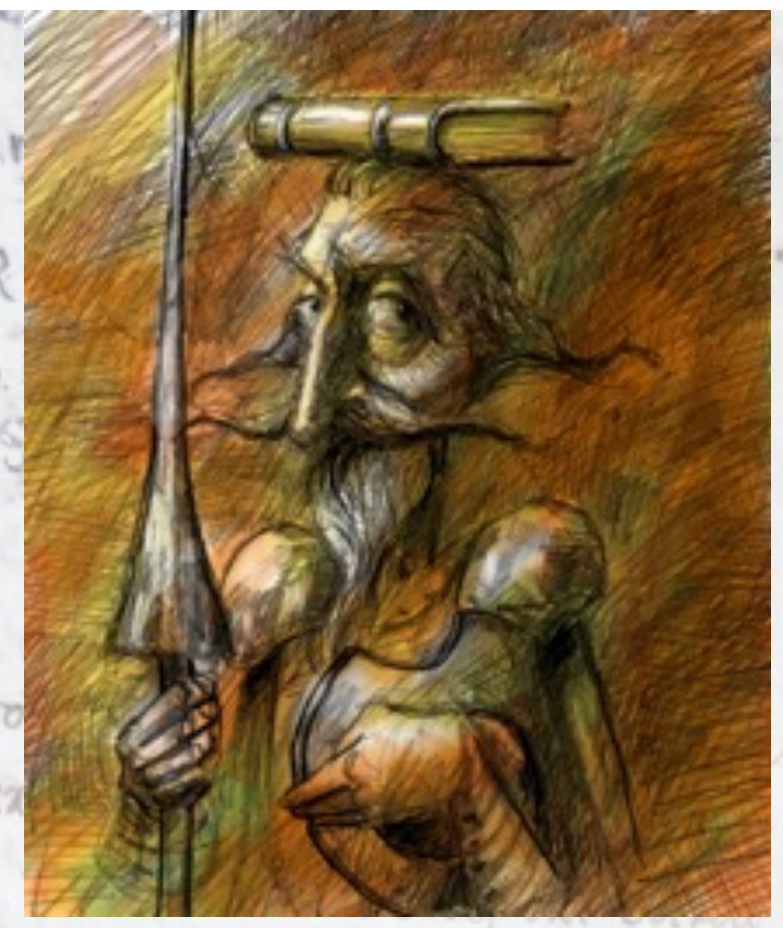

areabiteno entorndo entá Cama emfersi

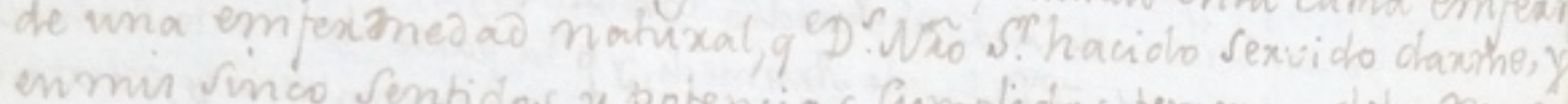
ére

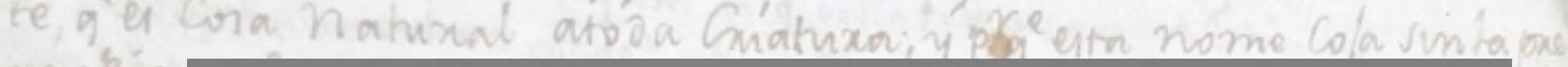
ventio creien SECCIÓN DE BUENA FUENTE midnot, $[\mathrm{dbf}]$

tabeno, Yolec: $C_{c}$

[Andrés Gattinoni] 


\title{
Richard Blackmore y su tratado sobre el spleen inglés*
}

\author{
Richard Blackmore and his Treatise on the English Spleen
}

ANDRÉS GATTINONI

\section{Resumen}

En 1725, el poeta y médico inglés sir Richard Blackmore publicó A Treatise of the Spleen and Vapours. Esta obra estaba dedicada a lo que el autor denominaba el "spleen inglés", un trastorno físico y mental, históricamente asociado con la melancolía que, según él, dominaba de manera universal y tiránica sobre los hombres y las mujeres de Inglaterra. Este artículo ofrece una edición crítica del prefacio del tratado de Blackmore. En la introducción, se presenta una semblanza del médico y se contextualiza el documento inscribiéndolo en dos debates de principios del siglo XVIII en los que participó sir Richard: la Querella entre los Antiguos y los Modernos y una polémica poco conocida acerca de la función fisiológica del bazo.

\section{Palabras clave}

Richard Blackmore - Spleen - Melancolía Anatomía - Antiguos y Modernos

\begin{abstract}
In 1725, poet and physician Sir Richard Blackmore published $A$ Treatise of the Spleen and Vapours. That workwas dedicated to what he called the "English Spleen", a mental and bodily disorder, historically linked with melancholy, which according to him had a universal and tyrannical dominion over the men and women of England. This article provides a critical edition of the preface of Blackmore's treatise. The introduction offers a biographical sketch of the physician and contextualizes the document by placing it within two debates of the early 18th century in which Sir Richard was involved: the Quarrel between the Ancients and the Moderns and a lesser-known controversy on the physiological function of the spleen.
\end{abstract}

\section{Key words}

Richard Blackmore - Spleen - Melancholy Anatomy - Ancients and Moderns

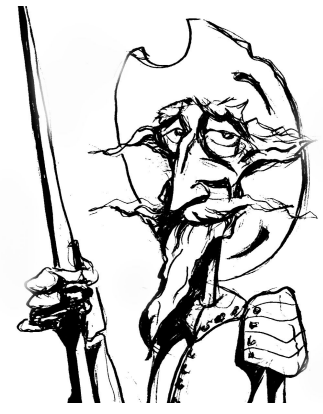

Recibido con pedido de publicación el 15 de mayo de 2018
Aceptado para su publicación el 20 de agosto de 2018
Versión definitiva recibida el 10 de octubre de 2018

Andrés Gattinoni, Universidad Nacional de San Martín, Consejo Nacional de Investigaciones Científicas y Técnicas, Argentina; e-mail: andres.gattinoni@unsam.edu.ar

\footnotetext{
* Agradezco los comentarios de los evaluadores anónimos

Esta obra se publica bajo licencia Creative Commons. Atribución-NoComercial-CompartirIgual (cc) EY-NC-SA 4.0 Internacional

Gattinoni, Andrés "Richard Blackmore y su tratado sobre el spleen inglés", Prohistoria, Año XXI, núm. 30, dic. 2018, pp.
} 241-264. 


\section{Introducción al documento}

1. "Si una tisis", decía sir Richard Blackmore (1654-1729) en el prefacio de $A$ Treatise of the Spleen and Vapours (1725), "es llamada con justicia por los extranjeros Tabes Anglica, o la tuberculosis inglesa, porque predomina mayormente y, de algún modo, es peculiar a este país, estoy seguro de que no hay menos razón para darle a la enfermedad que he elegido como tema de este tratado el apelativo del Spleen inglés". ${ }^{1}$ El escritor y médico ordinario de Guillermo III y Ana Estuardo, se sumaba así a una lista de observadores que habían destacado la prevalencia en Inglaterra del spleen, un trastorno físico y mental, históricamente asociado con la melancolía. ${ }^{2}$ En 1690, William Temple había evocado a "un gran médico extranjero" que había llamado al país "la región del spleen"3 ${ }^{\prime \prime}$, en 1712, Joseph Addison había escrito en The Spectator que "la melancolía es una especie de demonio que acosa nuestra isla". 4

Esta edición del prefacio de Blackmore a su tratado sobre el spleen y los vapores busca poner de relieve la difusión, incluso entre los médicos más destacados de la época, de la percepción de que esa enfermedad era un "mal inglés", como la llamaría George Cheyne unos años más tarde. ${ }^{5}$ En segundo término, la elección de esta fuente se debe a que sirve de puerta de entrada a dos debates de principios del siglo XVIII en los que participó Blackmore: la célebre Querella entre los Antiguos y los Modernos $^{6}$ y una polémica, menos conocida, sobre la función fisiológica del bazo.

\footnotetext{
${ }^{1}$ BLACKMORE, Richard A Treatise of the Spleen and Vapours: Or, Hypochondriacal and Hysterical Affections. With Three Discourses on the Nature and Cure of the Cholick, Melancholy, and Palsies, J. Pemberton, Londres, 1725, p. v. Todas las traducciones me pertenecen.

2 Véanse DOUGHTY, Oswald "The English Malady of the Eighteenth Century", The Review of English Studies, núm. 7, 1926, pp. 257-269; SENA, John F. "The English Malady: The Idea of Melancholy from 1700 to 1760", tesis doctoral, Princeton University, 1967 y COLBURN, Glen (ed.) The English Malady: Enabling and Disabling Fictions, Cambridge Scholars Publishing, Newcastle, 2008.

3 "...a great foreign physician called it, the region of spleen...", TEMPLE, William "Of Poetry", en The Works of Sir William Temple, Bart., vol. 3, J. Brotherton, Londres, 1770, p. 426.

4 "Melancholy is a kind of Daemon that haunts our Island", The Spectator No 387, 1712 en ADDISON, Joseph y STEELE, Richard The Spectator, vol. 5, J. M. Dent and co., Londres, 1898, pp. 256-257.

${ }^{5}$ CHEYNE, George The English Malady: or, A Treatise of Nervous Diseases of All Kinds, as Spleen, Vapours, Lowness of Spirits, Hypochondriacal, and Hysterical Distempers, Ec., George Strahan, Londres, 1733.

6 La bibliografía al respecto es abundante, véanse especialmente BURLINGAME, Anne Elizabeth The Battle of the Books in Its Historical Setting, B. W. Huebesch, Inc., Nueva York, 1920; FOSTER JONES, Richard Ancients and Moderns: A Study of the Rise of the Scientific Movement in Seventeenth-Century England, Washington University Studies, St. Louis, 1961 [1936]; LEVINE, Joseph M. "Ancients and Moderns Reconsidered", Eighteenth-Century Studies, Vol. 15, núm. 1, 1981, pp. 72-89; TINKLER, John F. “The Splitting of Humanism: Bentley, Swift, and the English Battle of the Books", Journal of the History of Ideas, Vol. 49, núm. 3, 1998, pp. 453-472; LEVINE,
} 
2. Richard Blackmore nació en 1654 en Corsham, Wiltshire, cerca de Bath. ${ }^{7}$ Hijo de un abogado adinerado, estudió en la Westminster School y luego en el St. Edmund Hall de Oxford. Allí conoció al célebre médico Thomas Sydenham (1624-1689), amigo de John Locke y veterano del ejército parlamentario en la guerra civil. Según una conocida anécdota, Blackmore habría preguntado al "Hipócrates inglés" qué autores debía leer para profundizar sus conocimientos de medicina y él le habría respondido: “Don Quijote, que es un muy buen libro; yo todavía lo leo". ${ }^{8}$ Hay distintas interpretaciones acerca de este relato, cuya veracidad, por otra parte, no es comprobable. ${ }^{9}$ En cualquier caso, Blackmore compartía con Sydenham una preferencia por la experiencia y la práctica por sobre la formación erudita. ${ }^{10}$ En esa época, los estudios médicos en las universidades inglesas consistían en un extenso período de lectura de textos clásicos y excluían casi todo conocimiento práctico, que el estudiante debía adquirir luego por su cuenta o como aprendiz de otro profesional. Siguiendo una trayectoria habitual desde el siglo XVI, Blackmore viajó al continente y obtuvo su doctorado en la prestigiosa Universidad de Padua, cuya tradición

Joseph M. The Battle of the Books. History and Literature in the Augustan Age, Cornell University Press, Ithaca, 1991; PATEY, Douglas Lane "Ancients and Moderns", en NISBET, H. B. y RAWSON, Claude (eds.) The Cambridge History of Literary Criticism, Vol. 4, Cambridge University Press, Cambridge, 1997; HARTOG, François Anciens, Modernes, Sauvages, Galaade, París, 2005.

${ }^{7}$ La siguiente síntesis biográfica fue elaborada a partir de JOHNSON, Samuel "Blackmore", en Lives of the English Poets, vol. 2, The Clarendon Press, Oxford, 1905 [1781], pp. 235-256. NOXON TOOMEY, Thomas "Sir Richard Blackmore, M. D. (1653-1729). An Account of his Life and Writings Drawn from Original Records not Known to his Earlier Biographers", Annals of Medical History, Vol. IV, 1922, pp. 180-188; BOYCE, Benjamin "The Dispensary, Sir Richard Blackmore, and the Captain of the Wits", The Review of English Studies, Vol. 14, núm. 56, octubre 1938, pp. 453-458. HUDSON LONG, E. "Notes on Sir Richard Blackmore", Modern Language Notes, Vol. 58, núm. 8, diciembre 1943, pp. 585-589; LANE FURDELL, Elizabeth "The Medical Personnel at the Court of Queen Anne", The Historian, Vol. 48, núm. 3, mayo 1986, pp. 412-429; GREGORI, Flavio "Blackmore, Sir Richard (1654-1729)", Oxford Dictionary of National Biography, Oxford University Press, Oxford, 2004. Disponible en: http://www.oxforddnb.com/view/article/2528. Consultado el 21/01/2015.

8 JOHNSON, Samuel "Blackmore”, cit., p. 236.

9 Véanse NOXON TOOMEY, Thomas "Sir Richard Blackmore...", cit., p. 181 y COOK, Harold The Decline of the Old Medical Regime in Stuart London, Cornell University Press, Ithaca, 1986, p. 185.

${ }^{10}$ Así, Blackmore diría: “...though Students should never read any Authors in Physick, but those that wrote in the Two last Centuries, they would want however no elder Writers to make them accomplished Physicians", BLACKMORE, Richard A Critical Dissertation Upon the Spleen, So far as Concerns the Following Question; Whether the Spleen is Necessary or Useful to the Animal Posses'd of It?, J. Pemberton, Londres, 1725, p. vii. 
educativa hacía énfasis en la filosofía natural aristotélica y las lecciones en el teatro anatómico. ${ }^{11}$

En 1687, Blackmore fue admitido en el Royal College of Physicians. Por entonces, la corporación londinense solo representaba a una fracción del mercado de la salud -integrado también por boticarios, cirujanos y barberos, entre otros, que tenían sus propios gremios - y no estaba a la vanguardia del conocimiento médico de la época. ${ }^{12}$ Sin embargo, a partir de un nuevo estatuto aprobado por Jacobo II, atravesaba un período de fortalecimiento que se expresaba en la duplicación del número de sus miembros - entre los cuales se incorporaron médicos graduados en universidades extranjeras y varios fellows de la Royal Society, lo cual estimuló una renovación intelectual- y en el endurecimiento de la persecución judicial contra quienes ejercían la medicina sin pertenecer al College..$^{13}$

Inicialmente, las relaciones de Blackmore con las autoridades de la corporación fueron tensas. La confrontación más concreta estuvo vinculada con el proyecto, formalizado en 1695, de crear un dispensario para proveer medicamentos gratuitos a los pobres de Londres. El College buscaba, de este modo, mejorar su imagen pública y perjudicar a los boticarios. Blackmore integró la facción que se oponía al dispensario. ${ }^{14}$ Esto tenía que ver con sus propias relaciones con algunos boticarios, pero en términos más generales con otro motivo de división al interior de la corporación.

Como varios de los miembros admitidos bajo el estatuto de Jacobo, Blackmore era un moderno. En medicina, esto implicaba una valoración mayor de la experiencia por sobre la erudición, una confianza en los descubrimientos recientes en anatomía que contradecían las enseñanzas de los antiguos, y la

\footnotetext{
${ }^{11}$ Sobre la educación de los médicos y esta trayectoria, véase COOK, Harold, The Decline..., cit., pp. 49-55. En cuanto a la escuela de Padua, véanse BYLEBYL, Jerome J "The School of Padua: Humanistic Medicine in the Sixteenth Century", en WESBTER, Charles (ed.) Health, Medicine and Mortality in the Sixteenth Century, Cambridge University Press, Cambridge, 1979, pp. 335370; SALVATORE, Filippo Antichi e Moderni in Italia nel Seicento, Guernica, Montreal, 1987; REBOLLO, Regina Andrés "A Escola Médica de Pádua: medicina e filosofia no período moderno", História, Ciências, Saúde - Manguinhos, Vol. 17, núm. 2, abril-junio, 2010, disponible en http://ref.scielo.org/fbkvr9. Consultado el 13/09/2018; VENTURA, Iolanda "Le lezioni inaugurali dei corsi di medicina dell'Ateneo di Padova tra Seicento e Settecento: appunti per una indagine tipologica", Quaderni per la storia della Università di Padova, Vol. 46, 2013, pp. 182-220.

${ }_{12}$ Sobre el mercado de la salud en Inglaterra, véanse COOK, Harold The Decline..., cap. 1 y PORTER, Roy Disease, Medicine and Society in England, 1550-1860, Cambridge University Press, Cambridge, 1993, especialmente caps. 2 y 4.

${ }^{13}$ Sobre el Royal College of Physicians en tiempos de Jacobo II, véase COOK, Harold The Decline..., cit., cap. 5, esp. pp. 206-207.

14 Sobre este episodio, véanse BOYCE, Benjamin "The Dispensary...", cit. y COOK, Harold The Decline..., cit., pp. 233-240.
} 
afición por espacios de sociabilidad novedosos como la Royal Society o las casas de café. ${ }^{15}$

Sin embargo, Blackmore también era moderno en otros sentidos. Además de médico, fue un escritor muy prolífico, autor de poesías, periódicos y panfletos políticos y religiosos. En 1695, publicó Prince Arthur, un poema épico modelado a partir de La Eneida que elogiaba a Guillermo III, que en dos años tuvo tres ediciones y una secuela. Esta obra le habría permitido ganarse el favor del monarca, quien lo hizo caballero y lo nombró como uno de sus médicos ordinarios (physician-in-ordinary) - cargo que conservaría durante el reinado de Ana -.16

Al mismo tiempo, el poema lo convirtió en objeto de las burlas de los wits, los escritores más destacados de su generación, quienes criticaron la tosquedad y monotonía de su estilo. ${ }^{17}$ Como varios de sus contemporáneos, Blackmore abogaba por una reforma de las costumbres en una cultura que consideraba inmoral e impiadosa. En sus textos, responsabilizaba por esa decadencia a los wits, especialmente a los dramaturgos, con quienes mantuvo enfrentamientos recurrentes a lo largo de su carrera literaria. ${ }^{18}$ En 1716 y 1717,

${ }^{15}$ COOK, Harold The Decline..., cit., p. 213. Johnson, quien admitía no haber leído los libros de medicina de Blackmore, decía que "By the transient glances which I have thrown upon them I have observed an affected contempt of the Ancients, and a supercilious derision of transmitted knowledge", JOHNSON, Samuel "Blackmore", cit., p. 250.

${ }^{16}$ Había dos tipos de médicos reales en la corte inglesa (a los que se sumaban los de la escocesa), el médico principal (first physician), que era uno solo, y los médicos ordinarios (physician-inordinary) que podían ser tres o cuatro. Véase COOK, Harold The Decline..., cit., apéndice 3.

${ }_{17}$ En un artículo clásico, Joseph Levine afirmó que el eje fundamental de la Querella entre los Antiguos y los Modernos en Inglaterra fue la contraposición entre las formas de acceder al pasado clásico de wits (antiguos) y scholars (modernos). Esto es, entre quienes privilegiaban la retórica, la imitación de los modelos clásicos y un tipo de polite learning, generalista y apto para la conversación, y aquellos que se acercaban a la antigüedad a partir de la erudición y el anticuariado. LEVINE, Joseph M. "Ancients and Moderns...", cit. El caso de Blackmore muestra que un moderno podía imitar a la poesía de los clásicos, rechazar la erudición y reivindicar un tipo de polite learning moralizante, al mismo tiempo que mostrar ese "affected contempt of the Ancients" mencionado por Johnson (véase nota ). Por ejemplo, en A Critical Dissertation Upon the Spleen, Blackmore se refería al trabajo erudito como "that inferior Kind of intellectual Endowments that consists in the Knowledge of Words and Languages, and not of Things" y agregaba "I detest the supercilious Airs, the Ostentatious Affectation and Dogmatical Pedantry that so often accompany this lowest Class of Scholars", BLACKMORE, Richard A Critical Dissertation..., cit., p. viii.

18 Véanse KRAPP, Robert Martin "Class Analysis of a Literary Controversy", Science \& Society, Vol. 10, núm. 1, 1946, pp. 80-92; BOYS, Richard Charles Sir Richard Blackmore and the Wits. A Study of "Commendatory Verses on the Author of the Two Arthurs and the Satyr against Wit", University of Michigan Press, Ann Arbor, 1949 y GAVIN, Michael The Invention of English Criticism: 1650-1760, Cambridge University Press, Cambridge, 2015, p. 61 y ss. La crítica de Blackmore a la inmoralidad del teatro tenía el antecedente de la "controversia de los escenarios" iniciada por Jeremy Collier en 1698. Véanse KRUTCH, Joseph W. Comedy and Conscience After the Restoriation, Columbia University Press, Nueva York, 1957 [1924]; ANTHONY, Rose The Jeremy 
Blackmore publicó en dos volúmenes sus Essays upon Several Subjects. ${ }^{19}$ Allí incluyó un ataque a Alexander Pope, supuesto autor de una parodia blasfema del salmo primero. ${ }^{20}$ Desde entonces, "everlasting Blackmore", como lo llamó Pope en The Dunciad, ${ }^{21}$ fue uno de los principales blancos del Scriblerus Club. Décadas más tarde, Samuel Johnson recordaría que el nombre del médico "fue empleado por tanto tiempo para referirse a cualquier epigrama de un escritor aburrido que finalmente se convirtió en sinónimo de desprecio". ${ }^{22}$

Bajo el reinado de Jorge I, Blackmore dejó de ser médico de la corte, pero su posición en el College of Physicians mejoró porque, en 1716, fue nombrado censor y elector. ${ }^{23} \mathrm{Al}$ año siguiente, el segundo volumen de su colección de ensayos incluyó uno titulado "An Essay Upon the Spleen", probablemente su primer texto dedicado a un tema médico. ${ }^{24}$ Allí argumentaba que el bazo no tenía ninguna utilidad para la preservación de la vida del individuo, sino que su función se relacionaba con la reproducción de la especie y, por eso, podía ser extirpado sin producir efectos adversos. Además, afirmaba que la enfermedad llamada vulgarmente spleen no derivaba de ese órgano, como creían los antiguos, sino que se debía a un trastorno en el sistema de los espíritus animales. Como se verá, el autor recuperaría ambos argumentos en su Treatise of the Spleen and Vapours.

Sus publicaciones médicas se multiplicaron después de 1722, cuando Blackmore renunció a su cargo en el College y se retiró a su casa de Boxsted, Essex. Es verosímil que solo entonces, luego de una prolongada práctica del

Collier Stage Controversy, 1698-1726, Marquette University Press, Milwaukee, 1937 y HUME, Robert D. "Jeremy Collier and the Future of London Theatre in 1698", Studies in Philology, Vol. 96, núm. 4, 1999, pp. 480-511.

${ }^{19}$ BLACKMORE, Richard Essays Upon Several Subjects, E. Curll y J. Pemberton, Londres, 1716 y BLACKMORE, Robert Essays Upon Several Subjects, Vol. II, A. Bettesworth y J. Pemberton, Londres, 1717.

${ }^{20}$ Ese poema fue uno de los motivos del enfrentamiento entre Pope y el librero Edmund Curll, véase BAINES, Paul y ROGERS Pat Edmund Curll, Bookseller, Clarendon Press, Oxford, 2007, p. 96 y ss.

${ }^{21}$ The Dunciad, II, 302 en POPE, Alexander The Major Works, Oxford University Press, Oxford, 2006, p. 482. Sobre la controversia, véase JEMIELITY, Thomas "A Mock-Biblical Controversy: Sir Richard Blackmore in the 'Dunciad'”, Philological Quarterly, Vol. 74, núm. 3, verano 1995, pp. 249-277.

22 "...was so long used to point every epigram upon dull writers that it became at last the byeword of contempt", JOHNSON, Samuel "Blackmore", cit., p. 252.

${ }^{23}$ El comité de censores se ocupaba de las actividades regulatorias del College, mientras que los ocho electores (elects) eran el gobierno de la corporación y quienes seleccionaban a los nuevos fellows. COOK, Harold The Decline..., cit., pp. 76-77.

${ }^{24}$ NOXON TOOMEY, Thomas "Sir Richard Blackmore...", cit., p. 182 cita como primer texto médico el Discourse upon the Plague de 1720, pero no tiene en cuenta este ensayo de 1717. No obstante, Blackmore distinguía entre ese texto, donde "he tratado el tema solo como un filósofo, que indaga en las obras y operaciones de la naturaleza", y el Treatise of the Spleen and Vapours en el que "he tratado el tema como un médico", BLACKMORE, Richard A Treatise..., cit., p. xxx. 
oficio, Blackmore se considerara preparado para dejar por escrito sus conocimientos médicos. ${ }^{25}$ Los últimos ocho años de su vida los pasó produciendo tratados sobre la plaga, la viruela, la tuberculosis, la gota, la hidropesía y el spleen. En esos textos, escritos en inglés y orientados a un público no estrictamente profesional, abordaba los temas desde una perspectiva pragmática, que profundizaba algunos rasgos ya iniciados por Sydenham y privilegiaba la experiencia y la observación sobre la especulación y la erudición.

3. En ese contexto, Blackmore escribió A Treatise of the Spleen and Vapours, que se publicó en 1725 y tuvo una segunda edición al año siguiente. ${ }^{26}$ El tratado estaba dividido en tres secciones. La primera abordaba la estructura y función del bazo, para luego estudiar la naturaleza de los vapores, sus síntomas y sus causas. La segunda, describía los métodos para curar la enfermedad y analizaba extensamente las afecciones histéricas y su tratamiento. ${ }^{27}$ La sección final se ocupaba sucesivamente de los cólicos, la melancolía y las parálisis. Por último, el impreso incluía una reedición del ensayo sobre el bazo de 1717.

El tratado estudiaba "este Proteo, esta enfermedad-postura", que se confundía con otras, pues estaba "acompañada por un largo tren de quejas y una triste variedad de sufrimientos". ${ }^{28}$ Entre ellos, el autor incluía síntomas físicos (indisposición y dolores estomacales, dificultades para la digestión que

\footnotetext{
${ }^{25}$ Esta es la interpretación de NOXON TOOMEY, Thomas "Sir Richard Blackmore...", cit., p. 186, que es compatible con la siguiente afirmación de Blackmore: "[...] I shall publish to the World only, at least chiefly, what Knowledge I have acquir'd upon this Subject by long Observation of the Nature of Diseases, and the Force of Medicine, and therefore I shall not oppress the Reader with numerous Citations from various Writers of Physick, nor transcribe their different and often inconsistent Notions, nor their endless Train of Medicines and Receipts too uncertain and precarious to be depended on", BLACKMORE, Richard A Discourse Upon the Plague, with a Preparatory Account of Malignant Fevers, John Clark, Londres, 1721, "The Preface", s/n.

26 A partir de una mirada superficial, la única diferencia que salta a la vista entre ambas versiones es que la segunda incluye al final A Critical Dissertation Upon the Spleen, que Blackmore había publicado en 1725 por separado. Por lo demás, las dos ediciones repiten incluso la misma fe de erratas, por cual probablemente se tratara de una reimpresión conjunta de los textos de 1725.

27 Blackmore, al igual que Thomas Willis y Thomas Sydenham, opinaba que la histeria era esencialmente la misma enfermedad que la hipocondría. Sin embargo, dice tratarlas por separado para seguir la costumbre. BLACKMORE, Richard A Treatise..., cit., p. 96. Véase JACKSON, Stanley W. Melancholia and Depression..., cit., cap. 11.

28 "...this Proteus, this Posture-Disease [...] is attended with a long Train of Complaints, and a sad Variety of Sufferings...", BLACKMORE, Robert A Treatise..., cit., pp. 16-17. El tópico de spleen como Proteo era un lugar común en la época. Anne Finch, por ejemplo, lo había llamado "Thou Proteus of abus'd Mankind" en su "Pindaric Ode to the Spleen" (1701), reproducido en INGRAM, Allan (ed.) Patterns of Madness in the Eighteenth Century. A Reader, Liverpool University Press, Liverpool, 1998, p. 59. Para una síntesis de las ideas del tratado de Blackmore, véase JACKSON, Stanley W. Melancholia and Depression..., cit., pp. 289-291.
} 
producían "tormentas de vientos hipocondríacos", ${ }^{29}$ trastornos en el colon, palpitaciones, temblores, desmayos, sudores fríos y dolores musculares), pero también síntomas en la cabeza "más variados y sorprendentes" como jaquecas, mareos, aturdimientos, "gran atontamiento y melancolía", insomnio, "a veces sueños tumultuosos, tristes y monstruosos", entre muchos otros. ${ }^{30}$ Sin embargo, a diferencia de otros autores contemporáneos, Blackmore no creía que la causa del spleen estuviera en el bazo, ni en otro órgano del hipocondrio, sino que derivaba de "los movimientos irregulares y desordenados de los espíritus y en la disposición irritable de los nervios". ${ }^{31}$ Distintos estímulos externos (impresiones violentas e inesperadas, noticias inoportunas, accidentes tristes, gritos repentinos o la mera apertura de una puerta) e internos (humores nocivos en el cerebro o la acción de fluidos ácidos o biliosos en los intestinos) podían perturbar y agitar los espíritus animales que circulaban por la "sensible y delicada constitución del sistema nervioso" y producir "movimientos desordenados y espasmos convulsivos y contracciones" de cualquier parte del cuerpo. $^{32}$

Se pueden destacar diversos aspectos del prefacio. En primer lugar, para justificar la relevancia del tema elegido, Blackmore resaltaba la frecuencia que tenían en Inglaterra las "afecciones hipocondríacas e histéricas, vulgarmente llamadas el spleen y los vapores". ${ }^{33}$ El nombre de "spleen inglés", decía, le parecía adecuado pues en ese país dominaba de manera universal y tiránica sobre ambos sexos. El autor incluso afirmaba que, si bien en los climas más cercanos al sol los trastornos de la mente y la imaginación eran muy frecuentes, el spleen inglés apenas se encontraba en habitantes de otros países. Esta idea ya había aparecido en su ensayo de 1717, donde Blackmore decía que "el spleen, una cualidad casi característica de esta nación, es de una naturaleza distinta a la locura y la enajenación, que son frecuentemente las enfermedades de los países

\footnotetext{
29 “...Storms of Hypochondriacal Winds...", BLACKMORE, Robert A Treatise..., cit., p. 17.

30 "...more various and surprizing [...] great Dulness and Melancholy [...] sometimes tumultuous, sad and monstruous Dreams...", BLACKMORE, Robert A Treatise..., cit., p. 23.

31 "...the irregular and disturbed Motions of the Spirits, and the irritable Disposition of the Nerves...", BLACKMORE, Robert $A$ Treatise..., cit., p. 11.

32 "...tender and delicate Constitution of the Nervous System [...] disorderly Motions and convulsive Spasms and Contractions...", BLACKMORE, Robert A Treatise..., cit., p. 30. Esta explicación se basa en las teorías de Thomas Willis, quien concebía el sistema nervioso en términos hidráulicos, como un conjunto de conductos huecos por los que circulaban los espíritus animales, sustancias muy sutiles que mediaban las comunicaciones del cerebro con el resto del cuerpo. Sin embargo, Blackmore introduce una diferencia, porque para Willis el bazo tenía un rol purificador de los fluidos corporales que, cuando fallaba, producía los efectos convulsivos del spleen. Véanse JACKSON, Stanley W. Melancholia and Depression..., cit., pp. 110115 y 281-284y SIMONAZZI, Mauro La malattia inglese. La melanconia nella tradizione filosofica e medica dell'Inghilterra moderna, Il Mulino, Bolonia, 2004, cap. 3.

${ }^{33}$ BLACKMORE, Richard A Treatise..., cit., pp. iii-iv.
} 
cálidos". 34 Luego, afirmaba que "quienes están dotados de una porción moderada de spleen en su complexión, son personas de un juicio superior y una vivacidad extraordinaria de su imaginación". ${ }^{35}$ Se trataba de la traducción, ya habitual para la época, del tópico peripatético del genio melancólico al lenguaje de los vapores. ${ }^{36}$ No obstante, el autor no explicaba por qué el temperamento inglés tenía esa mayor proporción de spleen.

Un segundo aspecto que vale la pena destacar del prefacio es la postura de Blackmore con respecto al debate entre antiguos y modernos. Si bien el texto no buscaba intervenir en la polémica abierta hacía más de tres décadas por William Temple, ni era una invectiva contra los wits que se habían burlado de sus incursiones literarias, el autor no podía dejar de sentar posición al respecto, lo cual da cuenta de la relevancia que tenían esas identidades en la cultura de la época.

Blackmore empleaba el lenguaje de los modernos. Como los filósofos de la Royal Society, se consideraba "un autor que aspira al beneficio general de la humanidad y quisiera extender la utilidad de sus obras a quienes las lean". ${ }^{37}$ Por lo tanto, escribía en inglés y no en latín, "con una expresión clara y obvia, no solo para los hijos del arte, sino para todas las personas inteligentes, aunque no sean grandes académicos o estudiosos de la medicina". ${ }^{38}$

Por otra parte, Blackmore criticaba duramente a los antiguos. Especial vehemencia tenía su reprobación de la influencia de Aristóteles en la medicina académica. Calificaba el pensamiento del filósofo como "vulgar y

\footnotetext{
34 "The Spleen, a Quality almost peculiar to this Nation, is of a different Nature from Madness and Distraction, which are frequently the Diseases of hot Countries...", BLACKMORE, Richard "An Essay upon the Spleen", en Essays upon Several Subjects, vol. II, W. Wilkins, Londres, 1717, pp. 166-237, aquí pp. 211-212.

35 "...those who are endow'd with a moderate Portion of the Spleen in their Complexion, are Persons of superior Sense and extraordinary Vivacity of Imagination...", BLACKMORE, Richard "An Essay upon the Spleen", cit., p. 212.

${ }^{36} \mathrm{Al}$ respecto, véanse KLIBANSKY, Raymond, PANOFSKY, Erwin y SAXL, Fritz Saturn and Melancholy. Studies in the History of Natural Philosophy, Religion and Art, Kraus, Nendeln, 1979; WITTKOWER, Rudolf y WITTKOWER, Margot Born Under Saturn. The Character and Conduct of Artists, New York Review of Books, Nueva York, 2007; BRANN, Noel L. The Debate Over the Origin of Genius During the Italian Renaissance, Brill, Leiden, 2002; MCMAHON, Darrin M. Divine Fury: A History of Genius, Basic Books, Nueva York, 2013; DIXON, Laurinda S. The Dark Side of Genius: The Melancholic Persona in Art, ca. 1500-1700, Pennsylvania State University Press, University Park, 2013.

${ }^{37}$ BLACKMORE, Richard A Treatise..., cit., p. vi.

${ }^{38}$ BLACKMORE, Richard A Treatise..., cit., p. vii. Sobre la apelación de los médicos a un público amplio en el marco de la polite society, véanse PORTER, Roy "Laymen, Doctors, and Medical Knowledge in the Eighteenth Century: The Evidence of the Gentleman's Magazine", en PORTER, Roy (ed.) Patients and Practitioners: Lay Perceptions of Medicine in Pre-Industrial Society, Cambridge University Press, Cambridge, 1985, pp. 283-314 y ARNAUD, Sabine On Hysteria: The Invention of a Medical Category between 1670 and 1820, University of Chicago Press, Chicago, 2015, cap. 3.
} 
descuidado", 39 carente de fundamentos en la razón o la experiencia, y reivindicaba la reciente revolución en la "república del saber" que "se liberó del yugo de la servidumbre y la intolerancia aristotélica" y afirmó el "derecho natural al libre ejercicio de la razón a partir de un examen imparcial de las cosas". 40

Blackmore se mostraba más indulgente con los antiguos previos a Aristóteles, como los "practicantes experimentales"41 que ejercían un arte médico simple antes del ascenso de la escuela peripatética. En otro escrito, se refería a ellos como "historiadores o registradores fiables de hechos". ${ }^{42}$ Sin embargo, esta reivindicación dependía de que sus observaciones fueran verificadas por estudios modernos. Así, el autor hacía referencia a los "experimentos de los antiguos" que, confirmados por "los testimonios coincidentes de los anatomistas modernos más eminentes", ${ }^{43}$ como Marcello Malpighi, probaban la inutilidad del bazo. En el mismo sentido, su mirada benévola hacia Anaxímenes de Mileto se vinculaba con la recuperación de su obra que había hecho Charles Le Pois para plantear la hipótesis de que todas las enfermedades derivaban de un déficit o deterioro del serum en el cuerpo.

Por último, la mayor parte del prefacio se ocupaba de la función fisiológica del bazo. La insistencia de Blackmore en este tema, sobre el que había escrito en su ensayo de 1717, es notable. No solo por la extensión que le dedicaba en el prefacio, sino por la inclusión del ensayo al final del Treatise of the Spleen and Vapours y la publicación simultánea de otro libro sobre el asunto: $A$ Critical Dissertation Upon the Spleen (1725). ${ }^{44}$ El motivo de esta redundancia parece ser "el reciente intento de un médico erudito y anatomista habilidoso de descubrir" 45 la función del bazo, agravado por el hecho "sorprendente" para Blackmore de que lo hiciera "sin refutar la evidencia que yo he producido para probar que tiene poca o ninguna utilidad con respecto al animal particular en el cual se encuentra". ${ }^{46}$

Blackmore no nombra al "ingenioso caballero" 47 que hizo este intento y no he encontrado alusiones a este debate en la historiografía. ${ }^{48}$ Entre la

\footnotetext{
${ }^{39}$ BLACKMORE, Richard A Treatise..., cit., p. viii.

${ }^{40}$ BLACKMORE, Richard A Treatise..., cit., p. ix-x.

${ }^{41}$ BLACKMORE, Richard A Treatise..., cit., p. ix.

42 "I pay likewise due Regard to the Physicians of the first Ages, as Historians or credible Recorders of Matters of Fact", BLACKMORE, Richard A Critical Dissertation..., cit. p. x.

${ }^{43}$ BLACKMORE, Richard A Treatise..., cit., p. xviii-xix.

${ }^{44}$ BLACKMORE, Richard A Critical Dissertation..., cit.

${ }^{45}$ BLACKMORE, Richard A Treatise..., cit., p. xvii.

46 BLACKMORE, Richard A Treatise..., cit., p. xviii.

47 BLACKMORE, Richard A Treatise..., cit., p. xvii.

48 Sobre debates anatómicos anteriores, véanse WEAR, Andrew Contingency and Logic in Renaissance Anatomy and Physiology, tesis de doctorado, Imperial College London, 1973 y WEBSTER, Charles "The Helmontian George Thomson and William Harvey: The Revival and
} 
publicación del ensayo de 1717 y la del Treatise of the Spleen and Vapours de $1725,{ }^{49}$ algunos autores ingleses habían escrito sobre el tema. ${ }^{50} \mathrm{Mi}$ hipótesis es que Blackmore se refería a William Stukeley (1687-1765), un médico y anticuario nacido en Lincolnshire, miembro de la Royal Society, quien en marzo de 1722 había dictado las Goulstonian Lectures del Royal College of Physicians. ${ }^{51}$ En ese ciclo anual de lecciones de anatomía, uno de los miembros más jóvenes de la corporación disertaba sobre un tema específico a partir de la disección de un cadáver. Stukeley dedicó su presentación, precisamente, al bazo y sus funciones. En su rol de censor y elector de la corporación, es posible que sir Richard haya presenciado las conferencias, que se publicaron al año siguiente bajo el título de Of the Spleen. ${ }^{52}$ En esa oportunidad, Stukeley había incluido también un reporte de la autopsia de un elefante que había realizado junto con Hans Sloane, presidente del College, lo cual justificaría el apelativo de "anatomista habilidoso" empleado por Blackmore. A ello habría que sumar que Stukeley había presentado su teoría como una recuperación del saber de Hipócrates, lo cual podría explicar en parte que Blackmore iniciara su Critical Dissertation con una extensa crítica al médico de Cos y a los eruditos contemporáneos, en el marco de la cual dudaba de "las reservas de

Application of Splenectomy to Physiological Research", Medical History, Vol. 15, núm. 2, abril 1971, pp. 154-167.

${ }^{49}$ Blackmore enfatiza que sus argumentos, aun "habiendo estado disponibles en el extranjero por varios años en mi Ensayo sobre el Bazo, citado anteriormente, ningún médico o anatomista ha intentado refutarlos" (BLACKMORE, Richard A Treatise..., cit., p. xx-xxi). Esto hace pensar que, efectivamente, se refiere a un texto posterior a 1717 (incluso, más cercano a 1725) y no a uno anterior que desconociera hasta hace poco.

${ }^{50}$ Entre esas fechas, se pueden mencionar:KEILL, James Essays on Several Parts of the Animal Oeconomy, G. Strahan, Londres, 1717; WOODWARD, John The State of Physick and of Diseases, T. Horne, Londres, 1718; QUINCY, Johm Pharmacopaeia Officinalis et Extemporanea; or A Compleat English Dispensatory, A. Bell y T. Vornam, Londres, 1718; QUINCY, John Lexicon PhysicoMedicum: or, A New Medicinal Dictionary, J. Osborn y T. Longman, Londres, 1719; MIDRIFF, John Observations Upon the Spleen and Vapours, J. Roberts, Londres, 1721. Entre estos autores, quien mejor se ajustaría a la descripción de "médico erudito y anatomista habilidoso" era James Keill. No obstante su libro, donde sostenía que el bazo era una cisterna que reducía la velocidad de la circulación de la sangre (Essays..., cit., p. 146) no solo no había sido publicado "varios años" después del ensayo de Blackmore, sino que era una reedición de su anterior An Account of Animal Secretion, G. Strahan, 1708, donde decía lo mismo (p. 45).

${ }^{51}$ Sobre Stukeley, véanse especialmente PIGGOTT, Stuart William Stukeley. An Eighteenth-Century Antiquary, Clarendon Press, Oxford, 1950 y BOYD HAYCOCK, David William Stukeley: Science, Religion and Archaeology in Eighteenth-Century England, Boydell, Woodbridge, 2002.

${ }^{2}$ STUKELEY, William Of the Spleen. Its Description and History, Uses and Diseases, Particularly the Vapors, with their Remedy. Being a Lecture read at the Royal College of Physicians, London, 1722. To which is Added Some Anatomical Observations in the Dissection of an Elephant, impreso para el autor, Londres, 1723. 
conocimiento, que algunos estudiosos escarbadores imaginan que han abierto en las elucubraciones del viejo". ${ }^{53}$

En Of the Spleen, Stukeley no solo no citaba a Blackmore, sino que además proponía una interpretación del bazo completamente opuesta a la suya. El anatomista argumentaba que ese órgano cumplía un rol primordial en la economía animal y que su mal funcionamiento era la causa del spleen. Stukeley, que era discípulo de Richard Mead y amigo personal de Isaac Newton, concebía al cuerpo humano como un microcosmos: una máquina maravillosa diseñada cuidadosamente por Dios. ${ }^{54}$ Preocupado, como varios de sus contemporáneos -incluido Blackmore-, por conjurar los posibles desvíos impiadosos de la nueva filosofía natural, Stukeley quería demostrar que no había nada inútil ni azaroso en la presencia del bazo en el diseño divino. ${ }^{55}$

Varios pasajes del prefacio pueden leerse como respuestas a los argumentos centrales de Stukeley. La premisa principal de Blackmore era que el bazo podía ser extirpado de cualquier animal sin perjuicio para su vida $y$, de allí, concluía que el órgano no podía tener ninguna utilidad para la preservación del individuo. El autor insistía una y otra vez en que su planteo

53 "...the Hoards of Knowledge, which some studious Delvers imagine they have broke open in the old Man's Lucubrations", BLACKMORE, Richard A Critical Dissertation..., cit., p. vi. La crítica de Blackmore no se limitaba al autor del estudio adverso sobre el bazo sino que apuntaba contra lo que consideraba un consenso de "the Generality of our Faculty" (p. iv). Por cierto, Hipócrates ya era reivindicado por su empirismo desde mediados del siglo XVII, entre otros, por Paracelso, Francis Bacon y Thomas Sydenham (SMITH, Wesley The Hippocratic Tradition, Cornell University Press, Ithaca, 1979, cap. 1). No obstante, cuando Blackmore atacaba al erudito moderno que "from the Greek Text he draws so many surprizing and important Inferences" (BLACKMORE, Richard A Critical Dissertation..., cit., p. v), posiblemente tuviera en mente a alguna otra persona. Por ejemplo, el newtoniano John Freind, a quien Stukeley se refería como "one who is now the ornament of our faculty" (STUKELEY, William Of the Spleen..., cit., p. 51). En 1717, Freind había publicado en Ámsterdam - pero con el sello de los censores del College of Physicians (incluido Blackmore) - un comentario sobre los escritos de Hipócrates acerca de las fiebres, que abrió una guerra de panfletos con John Woodward. FREIND, John Comentarii Novem de Febribus ad Hippocratis de Morbis Popularibus, Jansonio Waesbergios, Ámsterdam, 1717; GUERRINI, Anita "Isaac Newton, George Cheyne and the 'Principia Medicinae'” en FRENCH, Roger y WEAR, Andrew The Medical Revolution of the Seventeenth Century, Cambridge University Press, Cambridge, 1989, pp. 222-245, aquí p. 237.

${ }^{54}$ Sobre los médicos newtonianos, véanse SCHOFIELD, Robert E. Mechanism and Materialism. British Natural Philosophy in An Age of Reason, Princeton University Press, Princeton, 1970; GUERRINI, Anita "James Keill, George Cheyne, and Newtonian Physiology, 1690-1740", Journal of the History of Biology, Vol. 18, núm. 2, verano 1985, pp. 247-266; GUERRINI, Anita "Isaac Newton...", cit..

${ }^{55}$ Sobre la relación entre deísmo y filosofía natural, véanse FORCE, James E. "The Newtonians and Deism" en FORCE, James E. y POPKIN, Richard H. (eds.) Essays on the Context, Nature, and Influence of Isaac Newton's Theology, Kluwer Academic Publishers, Dordrecht, 1990, pp. 43-73 y HUNTER, Michael "Science and Heterodoxy: An Early Modern Problem Reconsidered" en LINDBERG, David C. y WESTMAN, Robert S. (eds.) Reappraisals of the Scientific Revolution, Cambridge University Press, Cambridge, 1990, pp. 437-460. 
estaba "más allá de toda controversia por experimentos repetidos", "innegables", "incontestables", y que nada podía "ser más claro". ${ }^{6}$ Esa reiteración podía deberse al estilo de "everlasting Blackmore" o al rencor provocado por el hecho de que su ensayo hubiera sido ignorado. No obstante, también podía relacionarse con que Stukeley había puesto en duda la veracidad de todos los reportes de experimentos que afirmaban haber extirpado el bazo de un animal sin alterar significativamente sus funciones vitales.

Por cierto, Blackmore no figuraba entre los testimonios estudiados por el anatomista, posiblemente porque en su "Essay upon the Spleen" de 1717 solo había referido experiencias de terceros. En el prefacio y en A Critical Dissertation Upon the Spleen, sir Richard decía haber removido el bazo de un perro, que luego vivió de manera saludable "por más de un año y mucho más, hasta donde sé" ${ }^{57}$ No obstante, su escueta relación difícilmente hubiera satisfecho los criterios de cuidado y paciencia que esperaba el médico de la Royal Society para considerar a un experimento verosímil. ${ }^{58}$

Por último, la premisa del diseño inteligente del cuerpo humano, que resultaba central para el argumento de Stukeley, según el cual el bazo no podía ser un tejido inútil, obligaba a Blackmore a repetir una precisión que había hecho en 1717. El órgano, decía, no tenía ninguna función con respecto a la preservación del individuo, de lo cual se seguía que debía desempeñar algún papel en la reproducción de la especie. Esta insistencia era necesaria para despejar sospechas de impiedad, pero el autor admitía no haber podido mostrar "claramente el modo en que esta víscera promueve la generación". ${ }^{59}$

Estos son solo algunos de los tópicos que pueden explorarse en A Treatise of the Spleen and Vapours y otros tratados médicos contemporáneos que se ocuparon de la supuesta epidemia de spleen en Inglaterra. La riqueza de este tipo de documentos no se agota en lo que ellos pueden decir sobre teorías fisiológicas perimidas. La reproducción de esta fuente es una invitación a fomentar una lectura cercana y contextualizada que permita entender mejor por qué una sociedad, en un momento específico de su historia, podía considerarse la exclusiva representante de una patología mental.

\footnotetext{
${ }^{56}$ BLACKMORE, Richard A Treatise..., cit., p. xxii, xxv, xxviii, xxix.

${ }^{57}$ BLACKMORE, Richard A Treatise..., cit., p. xx. La descripción de esta experiencia es incluso más breve en BLACKMORE, Richard A Critical Dissertation..., cit., p. 57.

58 "It's obvious to any that are acquainted with experiments to how much fallacy this is liable, how great care and judgement is necessary, beside patience, to make any observations of this kind, so as that they may be useful, and serve for a rational theory of the part...", STUKELEY, William Of the Spleen..., cit., p. 27.

${ }^{59}$ BLACKMORE, Richard A Treatise..., cit., p. xxix.
} 


\section{Nota sobre la edición}

En la transcripción se procuró preservar la puntuación, las contracciones, mayúsculas y bastardillas del original. Todas las notas son del editor.

BLACKMORE, Richard A Treatise of the Spleen and Vapours: Or, Hypochondriacal and Hysterical Affections. With Three Discourses on the Nature and Cure of the Cholick, Melancholy, and Palsies, J. Pemberton, Londres, 1725.

\section{The Preface}

If the Natives of this Island, either from the peculiar Constitution of the Air they breathe, or the immoderate Quantity of Flesh-Meats they eat, or of the Malt Liquors they drink, or any other secret Causes, are more disposes to Coughs, Catarrhs, and Consumptions, than the neighbouring Nations; they are no less obnoxious to Hypochondriacal and Hysterick Affections, vulgarly called the Spleen and Vapours, in a superior and distinguishing Degree. And of all the Chronical Distempers that afflict the Body, or disturb the Mind, these two, Consumptions and the Spleen, are in this Kingdom the most rife and prevalent; and either directly by their own Power, or by introducing other Diseases, make the greatest Havock and Destruction among the People. As by the various Kinds of Fevers, if Dr. Willis judges right, a third Part of the People are taken off; ${ }^{60}$ so it is reasonable to suppose that the like Proportion of those that escape the Fury of inflammatory and malignant Fevers, are destroyed or made miserable by the Diseases of which I am speaking. And therefore as I have published lately a Treatise on the Nature of Coughs and Consumptions, and the Method of Curing them, for the Service of my Countrymen; so in the same View I have now attempted to account for the Nature and Causes of the Spleen and

\footnotetext{
${ }^{60}$ WILLIS, Thomas "The Preface to the Treatise of Feavers" en Dr. Willis's Practice of Physick, Being the Whole Works of that Renowned and Famous Physician, T. Dring, C. Harper y J. Leigh, Londres, 1684,p. 45. Una apreciación distinta, pero que coincidía con el argumento general de Blackmore, era la de Thomas Sydenham quien calculaba que las fiebres constituían dos tercios de las enfermedades crónicas que aquejaban a las personas y las afecciones histéricas el tercio restante: "Hic morbus, si rectè calculum pono, Chronicorum omnium frequentissimè occurrit; \& sicuti Febres cum Appendicibus suis duas partes Tertias, ad Chronicos morbos simul sumptos si comparentur, complent, ita Affectus Hysterici (vel isti saltem quieo nomine insigniuntur) ad reliquae partis Tertiae dimidium assurgunt, hoc est, Chronicorum pars media sunt [...]", SYDENHAM, Thomas Dissertatio Epistolaris Ad Spectatissimum Doctissimumq; Virum Guglielmum Cole, M. D. de Observationibus Nuperis circa Curationem Variolarum Confluentium Nec Non de Affectione Hysterica, Walter Kettilby, Londres, 1682, p. 100.
} 
Vapours, and have set down the Method and Medicines, which in my Judgement are the most effectual for the Relief of the afflicted Patients.

If a Phthisis is justly called by Foreigners Tabes Anglica, or the English Consumption, because it is most predominant, and in a manner peculiar to this Country; I am well assured there is no less Reason to give to the Distemper I have chosen for the Subject of this Treatise, the Appellation of the English Spleen; since it has here gained such a universal and tyrannical Dominion over both Sexes, as incomparably exceeds its Power in other Nations: for though in foreign Climates, especially those nearer the Sun, Disorders of the Mind, Lunacy, and disturbed Imagination, are very frequent; yet the English Spleen, as I have now named it, and as I have described it in the following Pages, is comparatively but seldom found among the Inhabitants of other Countries.

An Author that aims at the general Benefit of Mankind, and would extend the Usefulness of his Works to all that read them, should reflect, that Perspicuity is a Perfection of Writing so necessary to this end, that without it the Sense and Meaning of the Author is in a great measure lost, even to attentive Readers; while the greatest Part of Men will not peruse, but lay by and reject those Writings, because of the Difficulty of understanding them; for Man by Nature is such a slothful Animal, that he cares not for Knowledge and Instruction, that is not communicated in an easy manner; such as gives him no Trouble, and requires but little Application. For this Reason I have endeavoured to convey my Sentiments to the Reader in clear and obvious Expression, not only to the Sons of Art, but to all intelligent Persons, though not great Scholars, or Students in Physick; and I hope there will not appear to Persons of a competent Capacity any thing intricate or obscure, for want of significant and intelligible Words; though sometimes it may happen otherwise, when the Subject-Matter it self is superior to their Apprehension, who have been wholly unacquainted with such Ideas.

Since all Diseases are Deviations from the natural irritate Rectitude of the Constitution; and therefore break the Symmetry, and subvert the regular Coherence and Subordination of the various Parts, they cannot but introduce the intestine Discord and Commotions, that tend to the Dissolution of the whole Structure: It is therefore reasonable to examine what Principles the human Body is compounded of, what Order, Connexion and Dependence is observed in a perfect Composition; that so Diseases that are the Errors or Defects of those Principles, and in some degree subvert their Harmony, due Mixture and Combination, may be discovered.

After Aristotle's ill-managed System, supported neither by Reason nor Experiment, had the good Fortune to become the Philosophy in Fashion, the Physicians generally gave into the Doctrine of his School, and formed their Notions of Diseases and their Cure, in Conformity to the Peripatetick Principles, 
that by a swift Growth acquired great Power and Authority: and now the Errors of this crude and lazy Philosophy being admitted into the Æsculapian Colleges, and mingling with their Conceptions, corrupted and depraved the Simplicity of the Art of Physick, as it was carried on before by the experimental Practisers. But at length a great Revolution happened in the Commonwealth of Learning, when the Authority of Aristotle, who had gained an Empire of vast Extend and long Duration over the Schools and Colleges of Knowledge, began to decline and grow out of Credit. And it is indeed wonderful that an Hypothesis of Philosophy should continue so long in great Reputation, that had only imaginary Foundations to rest upon. And now the Peripatetick Scheme began to be disgraced by many, that, from a generous Principle to assert their natural Right to the free Exercise of Reason, upon an impartial Examination of Things, threw off the Yoke of Servitude and Aristotelian Bigotry. And soon after, when the Colleges and Schools, that were defended by the Adherents of this Philosopher, had revolted and rebelled against the Prince of Science, which was then his Title, the greatest Part of the Heads of this Defection restored the Atomical or Corpuscularian Doctrine; ${ }^{61}$ and then the Physicians turning about with the Times, espoused this Hypothesis, and framed their Notions in Conformity to it.

But Carolus Piso, ${ }^{62}$ a French Doctor of considerable Reputation, endeavoured to revive the Philosophy of Anaxamenes, one of the first Masters of the Ionick School in Greece, ${ }^{63}$ who taught his Scholars, that Water was the fertile Parent of all compound Bodies; while others of equal Fame ascribed their Production to Air or Earth, or Fire; for each Philosopher had his favourite Element, on which he conferred the Honour of being the sole Principle that constituted all the Varieties of corporeal Beings. Now the Author I have named, in his Poem before his Book de Morbis Serosis, ${ }^{64}$ declares for the Doctrine abovementioned, that all Things are produced from Water; and therefore in Congruity to that Hypothesis, labours to shew in his Treatise, that Water, or

\footnotetext{
${ }^{61}$ Blackmore no formula aquí un juicio de valor negativo con respecto al atomismo. No obstante, en Creation: A Philosophical Poem (1712), su poema más aclamado, había refutado el atomismo de Epicuro y de sus seguidores modernos, y reivindicado las acciones de la Providencia. GREGORI, Flavio "Blackmore, Sir Richard...", cit.

${ }^{62}$ Charles Le Pois (1563-1633), médico de Nancy, Francia. En su Selectiorum observationum et consiliorum: De Cognoscendis et curandis, praecipue internis humani corporis morbis (1618) localizó el origen de la histeria en el cerebro.

${ }^{63}$ Se refiere a la escuela filosófica que surgió en Mileto a principios del siglo VI a. C. y que tuvo como principales exponentes a Tales, Anaximandro y Anaxímenes. Blackmore se equivoca al atribuir a Anaxímenes la idea de que el agua era la sustancia primera, origen de todas las cosas. Esa era la opinión de Tales. Para Anaxímenes, el principio de todas las cosas era el aire. Al respecto, aún resulta útil la obra clásica de VERNANT, Jean-Pierre Los orígenes del pensamiento griego, Paidós, Buenos Aires, 1992, caps. VII y VIII.

${ }^{64}$ Se trata de la obra citada de Charles Le Pois, que incluye un "Carmen Epicum Auctoris ad Livrum".
} 
Serum, in its defective and degenerate State, whether that consists in an excessive and disproportionate Quantity, or a depraved and putrefactive Quality, is the fatal Fountain whence Diseases of all Denominations are derived.

It is true, that this Notion is extended to an unreasonable Compass; for it is certain, that the ill Constitution either natural of acquired of the solid Parts, the relaxed Tone of the Fibres, the Glands and other organical Parts of the Body, or the Obstruction of the Minute Vessels by various Impurities and Concretions, are evidently the Causes of many and great Distempers; but if that Author had confined his Notion to Fluids, and charged the Production of the greatest Part of Diseases, to which Mankind are obnoxious, on the vitiated State of those Fluids, his Hypothesis might have been looked upon as very justifiable: Since it is reasonable to believe, that the Distempers that affect the Head, the System of the Nerves and the Animal Spirits, all proceed from the depraved serous Streams, that irritate and provoke the nervous Fibres, and drive the Spirits into Disorder and Confusion, as will appear at large in the following Pages.

But the Truth is, the ill Conformation to the solid Parts, and inordinate Dispositions of the Juices or Serum, often conspire as partial and confederate Causes in the Constitution of Distempers, and by affecting the Nerves, those irregular noxious Humours must have yet a wider Province, that appears at first sight. It is reasonable to suppose, that all kinds of Feavers [sic] take their Rise from the Nerves, since they all make their first Insult and Impressions on those Parts; as appears by the Rigours, Shiverings and convulsive Catchings and Tremblings, that introduce all kinds of this Disease, whether putrid or inflammatory; and when the Matter of the Distemper is discharged from the Nerves and received into the Blood, the Symptoms mentioned before, that belong to the Genus Nervosum in the milder Sort of Fevers, disappear, and are succeeded by a boiling Heat in the Blood, and usually a great Thirst. And it is very evident, that in Fevers attended with the greatest Putrefaction and Malignity, the Blood is not at all infected; but the destructive Matter convey'd by the Air, as it were a poisonous Gass, a pestilential Damp or a deadly Exhalation, like that which is sometimes let out by Delvers from metallick Veins, abolishes in a Moment all vital Motion, by extinguishing at once the System of the Animal Spirits: Thus in the Times of raging Plague, Persons walking in the Streets have fallen down dead in an Instant, as if struck with a Flash of Lightning; and others have lived but few Hours after they were attacked: whence it is plain, that this worst sort of Fevers affect the Spirits and Nerves only, since they destroy the Patient, before they can communicate, or produce any Symptoms there.

Now it is certain, that Hypochondriacal and Hyterick Patients very often suffer the same Symptoms that introduce a malignant Fever; and so much resemble each other, that they are not easily distinguished; and therefore they often impose even upon Physicians, as well as the Friends of the Patients, as I have observed in my Treatise on Fevers. 
While I agree with Piso, that the Diseases which affect human Bodies generally proceed from Depravity and ill Disposition of the Serum, or Fluids, distinguished from the Blood, I must explain my Meaning, which I suppose was the Sense likewise of the Author I have named; that is, that the Serum, which waters the Traces of the Brain, and passes through the Medulla Spinalis, and the minute Tracts of the Nerves, is not simple and unmixed elemental Water, but such as contains the generous and active Principles of refined Sulphur, Spirit, and volatile Salt, separated from the Blood by the Ministry of the Glands, or the fine Pores of the Brain; and then I conceive that the degenerate and vitiated State of such Serum is, according to the Opinion of our Author, the true Cause of most Diseases; and it is so, particularly of the Maladies I have discoursed upon in the following Treatise, will be shewn afterwards.

Since this Distemper has gone by the Name of the Spleen from the primitive Ages of Physick to this Time, one would think it reasonable to conclude, that some Defect in the Function of that Bowel had occasioned that Appellation; 65 but on attentive Enquiry into the Office of that Organ, it evidently appears to me, that it was not formed for the Benefit and Preservation of the Animal, of which it is a Part; and that therefore it is of no use at all in respect of the Individual. Physicians and Anatomists in all Ages, and in all Nations, have endeavoured to discover and settle the ministerial Duty of that organical Part; but notwithstanding the great Variety of Schemes laid down for a due Explanation of it, the Use of the Spleen is still a Secret that has eluded the Search of the most penetrating and sagacious Enquirers.

Nor does it continue less a Mystery, or involved in fewer Difficulties, since the late Attempt of a learned Physycian and skilful Anatomist to unfold it. ${ }^{66}$ It does not however cast any Dishonour on that ingenious Gentleman, that he has not found out the Use of the Spleen, which the greatest Physicians and Anatomists in all Ages have laboured in vain to discover; for those that undertake an impossible Task, are sure to miscarry. And that this is such, I conceive I have made evident, by demonstrating that the Spleen is of no use to any Individual that enjoys it, which I have done in my Essay on the Spleen. ${ }^{67}$ But that which is surprizing in his Conduct, is, that he should attempt, after this, to discover a new Way of accounting for the Usefulness of that Bowel, without

\footnotetext{
${ }^{65}$ Para Galeno, quien se apoyaba en Platón e Hipócrates, el bazo (en inglés, spleen) tenía la función de purificar la sangre, separándola de la bilis negra (o melancolía). Como señala Blackmore, la creencia en que una falla en ese órgano era una de las causas de la melancolía era la que fundamentaba que el término spleen fuera empleado para referirse a la enfermedad misma. Sobre estas explicaciones de la medicina clásica, véase JACKSON, S. W., Melancholia..., cit., $41-45$.

${ }^{66}$ Como se indicó en la introducción, este "learned Physician and skilful Anatomist" podría haber sido William Stukeley (1687-1765).

${ }^{67}$ BLACKMORE, Richard "An Essay upon the Spleen", cit.
} 
confuting the Evidence that I have produced to prove that it is of little or no Service at all in respect of the particular Animal in which it is found.

The Extirpation of the Spleen, without any consequent Mischief, is an uncontested Matter of Fact down to this Time from the eldest Ages, even before the Art of Anatomy or Dissection of human Bodies as well cultivated, and brought to such Degrees of Perfection as it afterwards acquired. And the Experiments of the Antients [sic] in this Case have been abundantly confirmed, by the concurring Testimonies of the most eminent modern Anatomists, particularly the celebrated Malpighius ${ }^{68}$ has recorded in his excellent Writings, many Instances of this Nature, in which himself was concerned, at least as a Director and an Eye-witness of the Operation.

If the Spleen then may be taken out entirely from the Bodies of Animals, and those Animals continue without any Marks of Injury or Diminution of Health in any respect whatsoever, but in the Possession of as full Vigour and Alacrity as before; then will it clearly follow, that this is an unnecessary or unuseful Organ of the Body, in respect to the Individual that is possessed of it: the Consequence is as clear as Noon-day. Now it evidently appears by repeated Experiments, as I have said, that the Spleen may be taken out of the Body, without the least Detriment to the Animal; and I my self have opened the Side of a Dog, and torn off with my Fingers the Spleen from the Parts to which it grew; yet without so much as tying up the Vessels, the Wound in the Side being sowed up, the Creature soon recovered, and shewed no sign of any Damage, or loss of Strength and Spirit, and continued in this healthful State more than a Year after, and much longer, as far as I Know.

It is in vain then for Anatomists to proceed in an obstinate Opposition to the clearest Evidence, and lose their Time and Labour in searching after the Use of the Spleen; which if the Arguments I have published are conclusive, is unnecessary to Individuals. And that my Arguments are unanswerable, I hence conclude, that they having been abroad several Years in my Essay on the Spleen, cited before, no Physician or Anatomist has attempted to disprove them:

\footnotetext{
${ }^{68}$ Marcello Malpighi (1628-1694) biólogo y médico boloñés, célebre por sus investigaciones de anatomía en las que empleaba el microscopio. Entre sus contribuciones se destaca su estudio del bazo, donde identificó la función del tejido conectivo de trabéculas que permean al órgano y también los folículos linfáticos que componen la pulpa blanca, conocidos como "corpúsculos de Malpighi". El trabajo de Malpighi era conocido en Inglaterra en buena medida por la correspondencia que mantuvo durante veinticinco años con la Royal Society, la cual también publicó obras del boloñés. Su influencia, junto con la de Nicolás Steno y Giovanni Alfonso Borelli, fue significativa para hacer viable el desarrollo de una medicina basada en la filosofía mecánica en Inglaterra. Véanse COOK, Harold J. The Decline..., cit., p. 195; BERTOLONI MELI, Domenico Marcello Malpighi: Anatomist and Physician, L. S. Olschki, Florencia, 1997; WILKINS, Bridget S. "The Spleen", British Journal of Hematology, núm. 117, 2002, pp. 265-274; ROMERO REVERÓN, R. "Marcello Malpighi (1628-1694), Founder of Microanatomy", International Journal of Morphology, Vol. 29, núm. 2, 2011, pp. 399-402.
} 
And therefore I cannot but think that they look upon it as too hard a Task; for if they judged themselves able, I have no Reason to believe they are unwilling to confute my Position. And if any learned Physician or Anatomist, that thinks he can invalidate my Reasoning, and subvert my Hypothesis, would do me the Favour to detect my Error, by shewing the unconvincing Weakness of my Arguments, I will give him sincere Thanks, and retract my Mistake: And it may be likewise be a Motive yet more prevalent with him to do this good Office, when he reflects, that by his clear and rational Discourse on this Subject, he may prevent others from falling into the same Error with my self, which they may be apt to do, since so much may be said in Vidication of my Assertion; for it is past all Controversy, from repeated Experiments, as before-mentioned, that the Spleen may be wholly spared; and that after Exsection, it will not be missed by the surviving Animal. And surely if it is so very unuseful to the Individual, that it neither contributes to the Preservation of his Health, Vigour and Vivacity, nor to the Beauty and Strength of its Structure, nor the Exercise of any one of its Faculties, we may then safely conclude, (for no Demonstration can be more evident) that this Bowel, like other organical Parts, contrived for Procreation, is insignificant and unserviceable to particular Animals; and then it follows undeniably from thence, that it must be formed and designed for some Office in promoting the Propagation of the Species; for no Man that acknowledges the Perfections of the Divine Author of Nature, can imagine than an intelligent Agent would act without an End; ${ }^{69}$ that he, that has appointed some Service and Use to the minutest Fibres and Glands of the Body, would contrive such an ample and admirable Organ, that takes up so much room in the Abdomen, and by its large Dimension and curious Fabrick, demands such Attention and Regard, should notwithstanding be subservient to no considerable Purpose whatsoever: and yet this must be the unavoidable Consequence, if it be not

\footnotetext{
${ }^{69} \mathrm{El}$ argumento de que el cuerpo es la obra de un autor divino e inteligente que no podría concebir un órgano sin un motivo era central en el debate sobre la función del bazo. Para William Stukeley, esa era la principal justificación de su teoría: "No one certainly that has but just dip'd into the anatomy of an animal body, and seen the amazing appearance of inimitable beauty, design and contrivance, thro' every minutest piece and member thereof, would imagine the spleen, boasting of a preheminence of structure, at least that it is inferior to none in the curiosity thereof, should be as it were a casual stroke and fortuitous job of almighty workmanship; but that it has its great uses equal in dignity and necessity with any other. And yet how many good Anatomists, after much pains and useless toil in its consideration, have as in a passion, at last concluded, it had no use or intention at all, and might as well have been omitted in the animal frame", STUKELEY, Of the spleen, cit., p. 25. Se trataba de un argumento en contra del ateísmo que ya había sido planteado por Richard Bentley en un conocido sermón predicado el 2 de mayo de 1694 en la iglesia Saint Martin in the Fields, como parte de las Boyle Lectures. Allí había dicho: "we cannot look upon it [the Finger] or the whole Body, wherein appears so much Fitness and Use, and Subserviency to infinite Functions, any otherwise than as the effect of Contrivance and Skill, and consequently the Workmanship of a most Intelligent and Beneficent Being", BENTLEY, Richard, The Folly and Unreasonableness of Atheism Demonstrated, H. Mortlock, Londres, 1693, sermón 3, p. 9.
} 
formed and intended for the End which I have named, since it must be destined either for the Service of the Species or the Individual.

It is impossible to inspect and contemplate this large Organ, without concluding, that it must have some important Office in the animal Administration and that it is not a superfluous and impertinent Fungus, or an Error or Sport of Nature; ${ }^{70}$ and then it will leave in the Mind no Ground or Reason of doubting, no not the least Hesitation about granting this Conclusion, that the Duty of the Spleen, which is little, if at all, concerned about the Preservation of the Individual, must almost wholly regards the Continuation of the Kind. If it be said, that there may be other Uses of the Spleen regarding the Individual, though they are not yet discovered by any Author; and that it is not just Arguing to assert, that there is no such Use, because it is unknown, which is the Case of Multitudes of Phænomena, nor that it is allowable for me to ascertain any Use meerly from this, that others have miscarried in their Attempts to discover it:

To this I answer, that I do not assert any Use of the Spleen, but on the contrary, have provided that it has none at all, as to the Individual, which is the Point in Controversy. And my Arguments are equally conclusive against all Uses of the Spleen, which Authors have hitherto ascribed to it, and any others whatsoever, that may be imagined to be yet undiscovered. For it is certain that the Spleen may be cut or torn from the Side, without the least Hurt to the Health of the Animal, the Truth of which is established by undeniable Experiments, as above-mentioned; then what can be more clear than this, that in respect of the Animal, whence it is taken, it is an idle or superfluous Organ; so that whatsoever Use may be supposed to be so hidden, that it eludes the Observation of the most penetrating and sagacious Men, it is plain it can have no Office or Function of any Moment or Importance to the Creature, which can live very comfortably, and perform all the Functions of Nature, as well without it as with it; it follows then from what has been said, that the Propagation of the Kind, by some way or other, was the End aimed at in the Formation of this curious Bowel, and by what Ways it contributes to it, I have expressed my Sentiments in my Essay on the Spleen, at the end of these Pages, to which I refer the Reader.

But here I must declare, that if I have not been successful, as I hope I have been, in explaining the Way and Method by which it contributes to Procreation; yet still I affirm, that whatever Use it has, it must regard the Continuation of the Species, and not the Individual: And therefore if I have not sufficiently unfolded the Manner how it is performed, yet my first and main Position is not affected by it; for still it must be allowed an undeniable and established Truth

\footnotetext{
${ }^{70}$ Nótese la similitud de esta frase con las de Stukeley y Bentley citadas en la nota anterior.
} 
from the unanswerable Arguments that enforce it, that the Use of the Spleen principally, if not wholly, respects the multiplying of Individuals.

These Arguments appeared so conclusive to several learned and judicious Gentlemen, that they told me, they looked upon my Hypothesis as established on good Reasons; nor have I heard any Objection made against it, except that one ingenious and learned Physician ${ }^{71}$ told me, he thought it was too much to build an Hypothesis upon a single Experiment. But this Insinuation, that I have really built an Opinion in Anatomy upon a single Experiment, must arise from Inattention or Disingenuity; the main Hypothesis I have advanced is, that the Use of the Spleen little concerns the Individual, and therefore it must respect the Species. To evince the Truth of the Proposition, I have alleged my own Experience, and cited others from the most accurate and skilful Anatomists, that sufficiently prove that this Bowel may be entirely spared, since the Animal feels no want of it in any Respect whatsoever: And if so, my Position stands unshaken, that this Bowel was formed for the Service of Generation, since it contributes little or nothing to the Benefit of Individuals; otherwise here is an eminent Bowel made for no Purpose, since it is neither useful to the Individual nor the Species. This is my Argument, founded on numerous and undeniable Experiments on various Animals; and the Way which I have suggested how the Spleen is subservient to Procreation, is likewise strengthened by Observations. How can then any Gentleman affirm, that my Notions are only supported by one Experiment? And what if this last Opinion had been built on no Observation or Experiment at all? Are these always wanting, even when scientifick Evidence may establish the Truth of any Proposition without them? And though it should be granted, that I have not clearly shewn the Way how this Bowel promotes Generation, yet that it does so, I have clearly proved by unanswerable Arguments; and so am at least in this Disputation on an equal Foot with the whole Profession, who believe that the Spleen is formed for the Service of the Individual, but are all ignorant how it performs its beneficial Office. And therefore the Truth of my main Position, that the Spleen is formed and contrived for the sake of the Species, does not stand or fall with that of my last Suggestion concerning the Way of its Usefulness; the first I mention as certain, the last only as probable; and each Conclusion is deduced from different Premises in my Argumentation.

Notwithstanding I have some Years ago published an Essay on the Spleen, as said before, together with other Miscellaneous Tracts; yet I have there treated the Subject only as a Philosopher, that enquires into the Works and Operations of Nature: and aiming at the Entertainment and Instruction of

\footnotetext{
${ }^{71}$ No resulta evidente a quién se refiere Blackmore en este caso. Sin embargo, la objeción que le atribuye a este "ingenious and learned Physician" es muy similar a la que algunas líneas más abajo le atribuye a otro "ingenious and learned Physician" que es posible identificar con George Sewell. Véase la nota siguiente.
} 
politer Readers, I have accommodated that Writing to their Taste, who are not so well pleased with grave and severe Disquisitions: But in these Pages I have managed the Subject as a Physician, and have said no more than what prepares my Way for laying down the Method of Cure. I have however added at the latter end the Dissertation before-mentioned; which, as it relates to the same Distemper, so it contains many Things that will give more Light into the Nature of it, and its various Symptoms; and so make this Performance more clear, and the System of my Notions more compleat. Though I have endeavoured to introduce little of that Writing into this, yet some Repetition of Words, Sentiments, and Matters that often occur, and are co-incident in this Treatise, and the Essay added at the end, being unavoidable in the Conduct I have observed, will, I imagine, be forgiven by the judicious Reader.

As to the three Discourses on the Cholick, Melancholy, and Palsies, that follow this Treatise on Hypocondriacal and Hysterick Affections, I shall not anticipate the Reader, by giving any summary Account of them in this Place. All that I shall say is this, that tho' I have not diffused and extended the Subjects to a great Length, by entering into the Consideration of many minute and less important Matters, that are fitter to swell a Volume, than to instruct and entertain the Reader; I hope, however, that I have set the Nature and Kinds of each of those Distempers, as well as the Methods of Cure, in so full and clear a Light, that he will find no Reason to complain of too great Succinctness and Brevity.

Since the writing of this Preface, I have received some Remarks from an ingenious and learned Physician, ${ }^{72}$ who did me the Favour to peruse it, for which I return him my hearty Thanks. That which is of the greatest Importance of his Observations, is, his Objection to my Position, That the Removal or Exsection of the Spleen may be performed, without destroying the Health of the Animal. If this, says he, were plainly demonstrated, I allow (and it would be

\footnotetext{
${ }^{72}$ Se trata del Dr. George Sewell (b. 1687-1726), según se desprende del siguiente pasaje de $A$ Critical Dissertation Upon the Spleen "...while my Treatise of the Spleen was in the Press, I received a Letter from Dr. Sewell, an ingenious and learned Physician, who tells me, that in his Judgement, the Evidence I have offered is not convincing and satisfactory...", BLACKMORE, Richard A Critical Dissertation..., cit., p. 2. Sewell era un médico y escritor nacido en Windsor que, debido a las dificultades que tuvo para sostener su consultorio, vendió su pluma produciendo poemas, traducciones y panfletos políticos, primero a favor de las políticas de Robert Harley y, luego, de su sucesor y adversario Robert Walpole. Véanse COURTNEY, W. "Sewell, George (bap. 1687, d. 1726)", Oxford Dictionary of National Biography, Oxford University Press, Oxford, 2004. Disponible en http://www.oxforddnb.com/view/10.1093/ref:odnb/9780198614128.001.0001/odnb9780198614128-e-25141 Consultado el 05/09/2018 y STARKIE, A. "Contested Histories of the English Church: Gilbert Burnet and Jeremy Collier", Huntington Library Quarterly, Vol. 68, núm. 1-2, marzo 2005, pp. 335-351.
} 
very strange it he should not) that the Spleen is unnecessary to the Individual. But then he declares, that in his Opinion the Arguments and Instances that I have brought in Evidence to establish the Truth of my Assertion, are not satisfactory; because, says he, 'there is required a greater Space of Time, and a long Series of convincing, repeated Experiments, to support my Hypothesis.' When I had deliberately reflected on this Remark, I continued settled in my Opinion, that I had made good my Allegation, by sufficient and unanswerable Reasons, and that I had not left any warrantable Ground of doubting about the Validity of my Proofs. And then I intended in this Preface to set the Matter in a stronger Light, and add an Answer to the Objection before-mentioned: But afterwards considering that I should not find room enough in a Preface, without swelling it into a very inordinate Size, to do this so fully and effectually, as not only to remove Objections and Difficulties from the Minds of candid and impartial Enquirer after Truth, but likewise to give them Satisfaction, who shall be the most indisposed to receive it, and thro' the Power of Prejudice of PartyPassions, have the greatest Reluctance and Displeasure to be undeceived and rightly informed, ${ }^{73}$ I resolved to publish a distinct Dissertation on this Subject, ${ }^{74}$ that is, that the Spleen may be safely taken out of the Bodies of Animals without any consequent Diseases, or any greater Alterations, than those that happen upon the Exsection of the Organs subservient to the Propagation of the Species, of which my Hypothesis makes the Spleen to be one.

\footnotetext{
${ }^{73}$ No es claro a qué "Party-Passions" se refiere Blackmore aquí. Si está discutiendo con William Stukeley, no sería probable que se tratara de diferencias políticas, pues ambos eran Whigs. El autor posiblemente aluda a diferencias entre los miembros del colectivo de médicos, por ejemplo, a la contraposición entre los médicos newtonianos como Stukeley y los que seguían el enfoque pragmático y anti-teórico de Thomas Sydenham, como Blackmore. Véase CUNNINGHAM, Andrew "Sydenham versus Newton: The Edinburgh Fever Dispute of the 1690s between Andrew Brown and Archibald Pitcairne", Medical History, Supplement núm. 1, 1981, pp. 71-98.

${ }^{74}$ BLACKMORE, Richard A Critical Dissertation..., cit.
} 